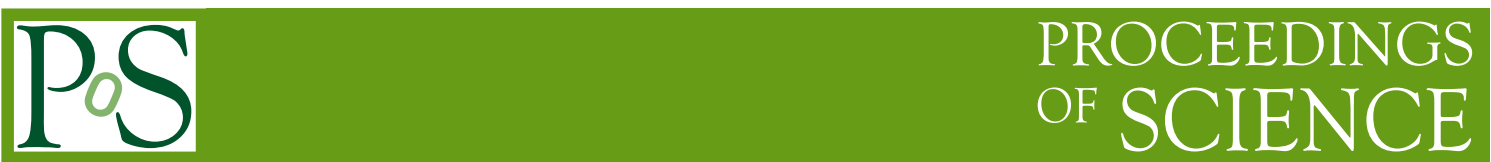

\title{
Complete Catalog of M31 Microlensing Events from MEGA
}

\section{Columbia University}

MEGA (Microlensing Exploration of the Galaxy and Andromeda) has compiled a catalog of candidate microlensing events from four telescopes (INT 2.5-meter, KPNO 4-meter, MDM 2.4 and 1.3-meter). The increment of new candidates over the INT-only sample is small. Furthermore, an HST imaging examination of the source stars in five of these candidates indicates that as many as two of them are background supernovae (although the other three are consistent with bona fide events). We are engaging in a similar HST investigation of the entire candidate sample, particularly those at large galactocentric distance. If the current rate of verification persists, this will correspond to a small halo lensing optical depth. We will conduct survey efficiency simulations to quantify this statement.

The Manchester Microlensing Conference: The 12th International Conference and ANGLES Microlensing Workshop

January 21-25, 2008

Manchester, $U K$ 
1.

References

[1] http://www.astro.columbia.edu/ alex/mega.pdf 